\title{
Estilo y redacción de trabajos académicos
}

\author{
Antonio Matas Terrón
}

Si me ofreciesen la sabiduría con la condición de guardarla para mí sin comunicarla a nadie, no la querría.

Lucio Anneo Séneca.

Nota: este texto es una revisión y modificación del capítulo 6 del libro del mismo autor "Introducción a la investigación en Ciencias de la Educación" disponible de forma gratuita en Bubok: $\quad$ https://www.bubok.es/libros/199453/Introduccio-n-a-la-investigacio-n-en-Cienciasde-la-Educacio-n

\section{Introducción}

El trabajo académico es un producto de la actividad del estudiante como aprendiz. El elemento básico que define cómo se debe redactar un trabajo académico son las indicaciones del docente. No obstante, y siempre en un segundo plano, es posible tener en cuenta otros elementos para la redacción. Por ejemplo, no cometer faltas de ortografías, utilizar correctamente los tiempos verbales, se precisos, claros y coherentes, etc.

El trabajo académico, como le ocurre a la inmensa mayoría de documentos científicos, se rigen por su propio estilo literario, alejado de otros estilos como la novela o la poesía. En cualquier caso, debe tenerse en cuenta que el estilo literario científico evoluciona con el tiempo. Así, resulta lalmativo leer trabajos científicos del pasado comparándo las expresiones con la actualidad. En cualquier caso, es de suponer que el estilo seguirá evolucionando.

En este enlace se puede cotejar el estilo literario de Ramón y Cajal: https://cvc.cervantes.es/ciencia/cajal/cajal_articulos/hormigas.htm. 
Además, en la literatura científica no es sólo suficiente como se redacta, sino también los medios de su difusión. Existen distintos tipos de informes científicos que pueden clasificarse de diferentes formas, según utilicemos un criterio u otro. Por ejemplo:

- Longitud del trabajo: Breve (artículo, comunicación, ponencia) o extenso (tesis, monografía de investigación, ensayo).

- Audiencia: Técnico (dirigido a personal con capacitación suficiente para entender la jerga de la disciplina) o divulgativo (dirigido al público en general).

- Soportes: Escrito u oral. Actualmente a éstos dos medios hay que añadir el soporte digital.

- Paradigma de referencia: empírico-analítico o cualitativo (interpretativo, investigación-acción, etc.).

Esta tipología también se puede generalizar a los trabajos académicos.

A continuación vamos a comentar tres formas de realizar el informe, basándonos en el último criterio apuntado en el paradigma de referencia.

\section{Trabajos de tipo cuantitativos}

La estructura de estos trabajos viene marcada generalmente por estándares propuestos por las asociaciones de investigación. Uno de los estándares es la normativa APA (American Psychological Association). Según esta normativa la estructura de este tipo de informe es la siguiente:

- Título

- Resumen

- Introducción

- Resultados

- Discusión

- Referencia

- Apéndices

- Tablas

- Encabezamientos de figuras 


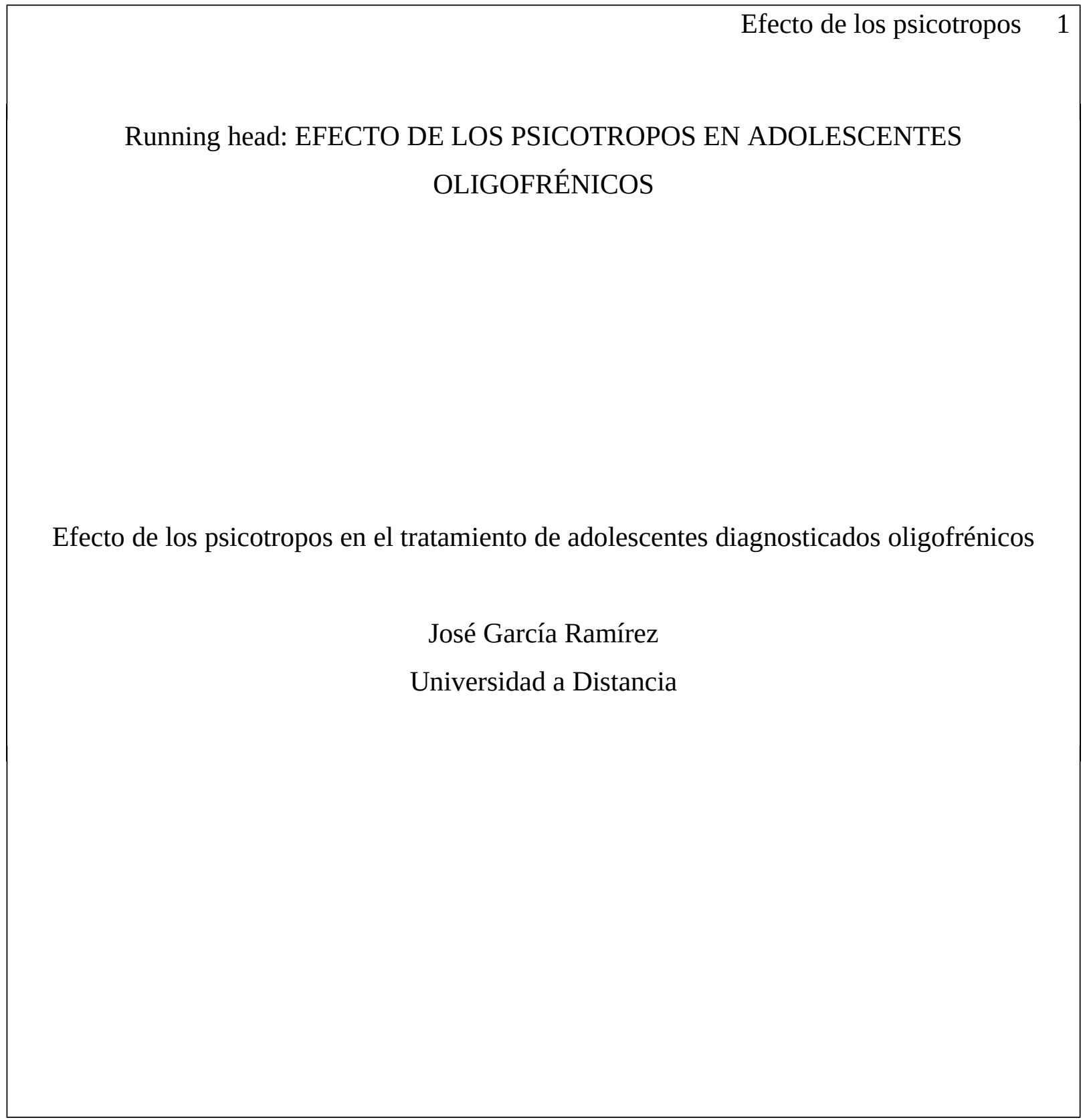

Ilustración 1. Primera página del informe según normativa APA

Según el estilo APA, en la primera página del informe se escribe el título, el autor o autores, la institución a la que pertenece cada autor y un encabezamiento (ilustración 1):

- Título: Debe tener una longitud de entre 10 y 12 palabras como máximo y estar centrado en la página. El título debe ir en minúsculas. En la parte superior derecha de la página se escribe el título acortado (running head) en mayúsculas, 
con una longitud máxima de 50 caracteres. Este título corto es obligatorio solamente cuando el informe se pretende enviar a una revista.

- Autor o autores: Se escriben debajo del título, centrados y en minúscula.

- Afiliación: Bajo los autores se indican las entidades a las que pertenecen los autores. También se redacta en minúscula y centrado.

- Encabezamiento: El encabezamiento se alinea a la derecha de la página. Incluye el título abreviado, cinco espacios blancos y el número de página.

El resumen se escribe en la segunda página. Se inicia con el título "resumen" o "abstract" dependiendo el idioma en que se escriba en informe. El título del epígrafe se escribe centrado, mientras que el cuerpo de texto se alinea a la izquierda. El resumen debe tener una longitud máxima de entre 120 y 150 palabras. Debe incluir el problema de investigación, los participantes, el método utilizado, los resultados principales obtenidos y la conclusión principal (ilustración 2).

Autoestima y reactividad 2

Resumen

El objetivo de este estudio es analizar la existencia de una relación entre diferentes conceptos y el fenómeno de reactividad. Las variables que se estudian son: ansiedad, deseabilidad social, autoestima, ansiedad psicológica, ansiedad motora, y ansiedad cognitiva. Se construyó un instrumento específico (llamado retoma). Se contó con una muestra de 180 personas voluntarias, alumnos de Pedagogía. Los resultados muestran una relación significativa entre la autoestima y la reactividad.

\section{Ilustración 2. Resumen según la normativa APA}

En la siguiente página (tercera del documento) se escribe la introducción. Se trata de una descripción general del tema de investigación, incluyendo una revisión de trabajos previos 
relevantes realizados por otros investigadores. Dentro de la introducción debe exponerse el objetivo u objetivos de la investigación, las variables utilizadas y las hipótesis planteadas. La introducción comienza a escribirse directamente, sin ningún título de epígrafe.

Después de la introducción se redacta el epígrafe "método", donde se detallan las características de la muestra (edad, género, lugar de procedencia, etc.). La palabra "sujeto" se utiliza principalmente para referirse a animales, mientras que para el estudio de seres humanos se utiliza la palabra "persona".

En este apartado también se describen los instrumentos de recogida de datos, detallando sus características y el papel que han jugado en la investigación. Se incluye a continuación el procedimiento seguido, donde indicaremos el tipo de investigación realizado, las fases que se han seguido, la organización de las variables independientes, etc. También podemos incluir cualquier otra cuestión que podamos considerar de interés para la comunidad científica y que facilite la comprensión del estudio.

\section{Niveles de titulación:}

Nivel 1:

\section{TITULO EN MAYÚSCULAS CENTRADO}

Nivel 2:

Título en Mayúsculas y Minúsculas Centrado

Nivel 3:

\section{Título en Mayúsculas y Minúsculas Centrado y en Cursiva}

Nivel 4:

Título Secundario en Mayúscula y Minúsculas en Cursiva y Alineado a la Izquierda Nivel 5:

Encabezado de párrafo con sangría, en minúsculas, en cursiva, alineado a la izquierda y que finaliza con punto.

\section{Ilustración 3. Niveles de títulos según APA}

El epígrafe se inicia con el título "Método" centrado y en minúscula. Se pueden indicar subepígrafes (participantes, instrumentos y procedimientos) con el título en minúscula y alineados a la izquierda. 
En el siguiente apartado se incluyen los resultados obtenidos en los análisis realizados. Generalmente se comienza indicando los resultados descriptivos, posteriormente los correlacionales (si se han realizado), y por últimos los inferenciales, multivariantes, etc. En el apartado de "Discusión" se exponen las aportaciones más relevantes de la investigación, las implicaciones que conllevan, y se concluye comentando las hipótesis propuestas así como posibles líneas de estudio (esto último se realiza de forma muy breve).

La referencias bibliográficas también se redactan siguiendo los estándares propuestos por la APA (ver anexo).

\section{Trabajos cualitativos}

Dentro del enfoque cualitativo nos encontramos una gran diversidad de formas de realizar los informes de investigación. Esto es debido a que la elaboración de un informe de este tipo es muy flexible y no se rige por reglas ni estándares claramente aceptados por la mayoría de los investigadores.

Un informe interpretativo podría organizarse en los siguientes apartados:

a) Introducción:

- Descripción del contexto general.

- Panorámica general del tema en la literatura especializada.

- Exposición del propósito del informe y la partes que lo componen.

b) Bases de la investigación:

- Intereses y expectativas de los investigadores, lo que permite valora la influencia de los mismos en el proceso.

- Cuestiones que se pretenden resolver justificando por qué se afrontan desde esta perspectiva.

- Formación y experiencia del equipo investigador.

c) Contexto de investigación:

- Localización de los informantes: Aspectos geográficos, demográficos, históricos, proceso se selección. 
- Escenario de investigación y conocimiento previo del mismo por parte de los investigadores.

d) Proceso de investigación:

- Descripción y justificación de los métodos, así como de los cambios realizados en los mismos a lo largo del proceso.

- Rol de los investigadores y relación con los informantes para comprender la interacción en la recogida de información.

- Evolución de los objetivos o cuestiones a resolver.

- Especificación de los sistemas para recoger los datos.

- Proceso de análisis de la información.

- Sistema de información a los participantes sobre los resultados.

e) Conclusiones:

- Indicar las distintas deducciones, consecuencias, corolarios, etc., que han ido apareciendio a lo largo de la investigación.

- Consecuencias de la investigación sobre el contexto y nuevas perspectivas que se abren.

En el caso de la investigación-acción (como ejemplo dentro del paradigma sociocrítico) el informe tendrá que contemplar una perspectiva temporal. Se trata de poner de manifiesto la evolución, exponiendo los procesos de acción y la evaluación llevada a cabo. 
Introducción

Análisis del contexto

Fase de planificación

Proceso de desarrollo

Reflexión y evaluación

Bibliografía Anexos Cuadro 1. Apartados y

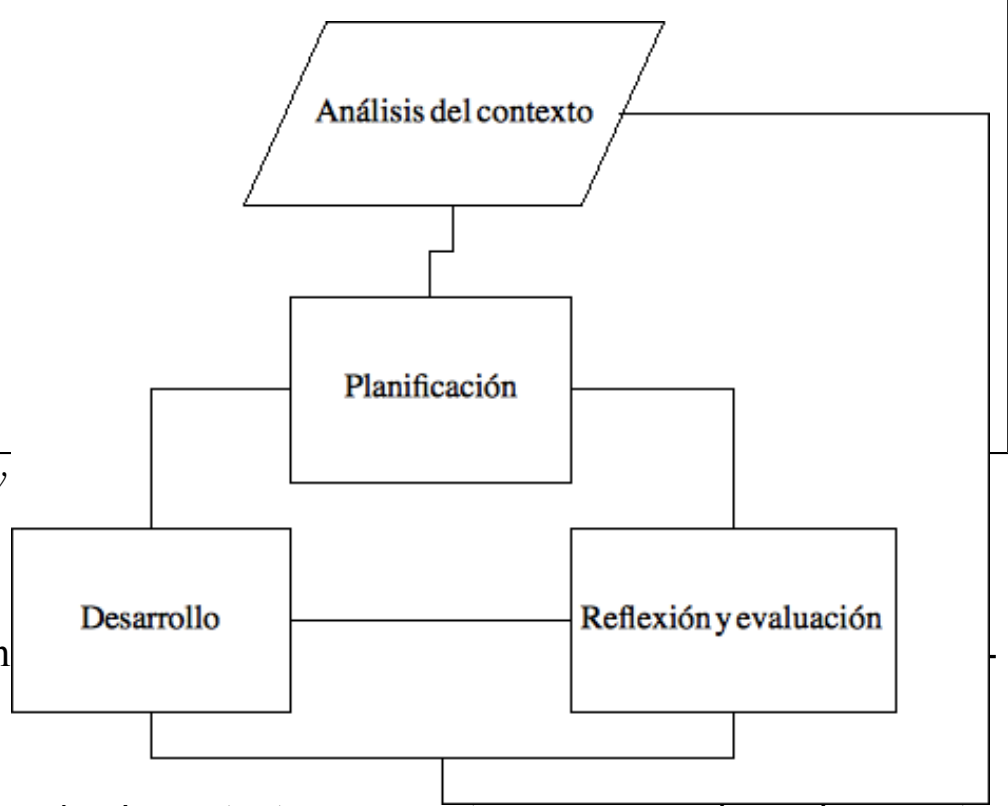

- Introducción: Descripción de contextos, circunstancias que rodean el proyecto, conceptos iniciales, etc.

- Análisis del contexto: Descripción pormenorizada del contexto (histórico, geográfico, demográfico, etc.), proceso de constitución del equipo de trabajo (datos profesionales, posición dentro del grupo), objetivos y motivaciones del equipo, papel de los investigadores. Así como la identificación y descripción del tema a investigar (antecedentes, motivos para abordarlo, percepción de los participantes, etc.).

- Fases del plan de acción: Descubrimiento del problema (como surge y como evoluciona) y planificación de las estrategias o hipótesis de acción.

- Desarrollo del proceso: Cómo se organiza el trabajo en el grupo, puesta en marcha de las actividades y proceso de recogida de datos.

- Reflexión y evaluación: Análisis de la información, interpretación de los resultados (a través de la reflexión) dentro del contexto, explicación de los procesos para garantizar la credibilidad y la validez (triangulación por ejemplo), 
conclusiones (efectos de la acción, efectos en las personas participantes, aportaciones teóricas y prácticas al ámbito social y de conocimiento), así como la comunicación de los resultados a los investigadores y participantes para proponer nuevas vías de acción

- Bibliografía y anexos.

\section{Aspectos a considerar en la redacción}

El discurso científico debe plasmarse también en su producción literaria. El estilo científico se caracteriza por ser claro, preciso y ordenado. El orden permite pasar de forma natural de una idea a otra. Esto se consigue utilizando los conceptos adecuados para cada idea, manejando las puntuaciones de forma correcta, recurriendo a las palabras justas y llamando a cada cosa por su nombre. Algunas sugerencias para ordenar las ideas son las siguientes:

- Agrupar las ideas en bloques.

- Desarrollar cada bloque.

- Conectar los bloques a partir de un criterio fijo, por ejemplo a partir del desarrollo cronológico, de la causa-efecto, o de la comparación entre ideas.

Para ser preciso en el discurso se pueden seguir los siguientes consejos:

- Prescindir de los sinónimos. No utilizarlos para referirse a las variables.

- Intentar que el texto sea breve, se desaconsejan frases de más de 20 palabras. Lo deseable es que cada frase contenga una sola idea.

- Evitar expresarse solamente con jerga técnica ${ }^{1}$ si no es necesario.

- Utilizar el pasado verbal para la revisión de la literatura, descripción del procedimiento y resultados. Para el apartado de discusión es preferible utilizar el presente. Siempre deben evitarse los cambios de tiempo en una misma sección o epígrafe.

\footnotetext{
1 Debe tenerse en cuenta que el lector puede no ser un experto en la materia, o bien estudiantes que se están iniciando en la disciplina, o incluso personas con iniciativa que han sentido curiosidad por el tema.
} 
- También es recomendable evitar utilizar palabras innecesarias (plenasmos). Por ejemplo, es mejor usar "fueron iguales" que " los dos fueron iguales", "50 participantes" en lugar de "un total de 50 participantes", "cuatro grupos" en lugar de "cuatro grupos distintos", etc.

- Evitar expresiones coloquiales que pueden ser imprecisas en otro contexto (la mayoría, casi todos, etc.).

- Evitar el antroporfismo. Usar por ejemplo "a través del experimento demostramos que..." en lugar de "el experimento demuestra que....", "en la tabla se indica que..." en lugar de "la tabla indica que ...", etc.

Abreviaturas más utilizadas en los textos científicos:

c. f.: compárese i. e.: esto es, es decir

e. g.: por ejemplo et al.: y otros

viz: es decir, esto es etc.: y así

vs.: contra, en oposición a

Cuadro 2. Abreviaturas habituales

\section{Presentación de los resultados}

Como se apuntó al principio, uno de los criterios de clasificación de los informes es el soporte que se utiliza para su presentación (oral, escrito o digital). En todas las modalidades el informe adquiere unas características específicas. En el caso oral los pasos recomendables para preparar una exposición son los siguientes:

- Preparar por escrito un borrador del contenido y pedir a algún compañero que lo valore críticamente.

- También por escrito, preparar una versión final una vez revisada la anterior.

- Estimar el tiempo que se utilizará para a cada apartado de la exposición y tratar de ajustarlo al tiempo del que disponemos.

- Realizar el material audiovisual de apoyo. Este material debe coordinarse con la exposición. 
- Ensayar la exposición completa con todos los recursos que se utilizarán (transparencias, audios, vídeos, etc.).

- Anticipar posibles consultas.

- Preparar algún material escrito para entregarlo durante la exposición, si se considera que éste puede ayudar a la comprensión y seguimiento de la misma.

Durante la exposición es aconsejable:

- Comenzar con una presentación personal si nadie la ha realizado previamente por nosotros.

- Indicar de qué se va a hablar y cuanto tiempo aproximado durará la intervención.

- Seguir el manuscrito (o el esquema del mismo) realizando las improvisaciones que se consideren oportunas en cada momento, con el fin de facilitar la compresión de las ideas expuestas, animar la intervención de los asistentes, etc.

- Terminar con una síntesis de la exposición.

- Permitir preguntas y responderlas con brevedad y con claridad. Si alguna pregunta no se sabe responder, se puede decir que en ese momento "no se está en condiciones de contestarla".

Junto con todo esto, durante la exposición es recomendable seguir una serie de normas comportamentales y gestuales, que suelen aliviar la tensión del momento y ofrecer una sensación de cercanía entre la audiencia y el ponente. Algunas de ellas son las siguientes:

- Intentar mantener contacto visual con el público.

- Usar anécdotas para amenizar la exposición.

- Fomentar la participación de los asistentes.

- Evitar las muletillas al exponer. Las muletillas como “¡eh!”, “¿verdad?”, “¿no?”, “este...", “vale?”, etc. No solamente son molestas, sino que acaban por distraer la atención del oyente. 
- Ser fluido en la exposición, utilizando un lenguaje correcto, tiempos verbales coherentes, y un vocabulario adecuado al nivel cultural medio de la audiencia.

- Usar adecuadamente el lenguaje corporal para ayudar a la presentación.

- Ajustarse al tiempo disponible.

Las presentaciones pueden adquirir distintos formatos, así en las formas orales encontramos las ponencias, comunicaciones, la parte expositiva de las tesis, y la parte expositiva de los pósteres.

Con relación a los soportes escritos, tanto en papel como digitales, destacan los artículos, las monografía, y las tesis. El proceso para redactar un documento escrito es el siguiente:

- Organizar en un esquema las ideas principales y las secundarias, estableciendo la relación que mantienen entre unas y otras.

- Agrupar por bloques los conceptos o ideas que están estrechamente relacionados entre sí.

- Ordenar la agrupación anterior en epígrafes y subepígrafes, tratando de ajustarse a una normativa estilística, si así nos lo requieren.

- Comenzar a redactar siguiendo el esquema creado. Durante la redacción debe cuidarse el vocabulario y la ortografía. Para conectar las distintas ideas entre sí, debemos construir adecuadamente las estructuras subordinadas, usando conectores que garanticen la comprensión del lector: cronológicos (más tarde, al poco tiempo, superado el tiempo, etc.), espaciales (detrás, junto con, etc.), de orden (tras..., a continuación, finalizado..., etc.), o de razonamiento (...implica, por lo tanto, etc.).

- Revisar el escrito. Siempre es bueno pedirle a otras personas que revisen críticamente el texto, tanto desde el punto de vista formal (estructura, ortografía, claridad del escrito, etc.) como de contenido (plausibilidad de las propuestas, consistencia del estudio, etc.).

- Reescribir el texto para corregir las deficiencias y errores. 
Con relación a los formatos más habituales para presentar una investigación tanto por escrito como oral, son los siguientes:

- Monografía: Es un trabajo que versa sobre un tema único que suele ser tratado brevemente, pero en profundidad. A veces, se suele asociar al resultado de una revisión bibliográfica y no tanto al trabajo de campo, si bien, este criterio no siempre es cierto para todas las monografías. De hecho, el término monografía es bastante flexible, siendo habitual encontrarlo asociado a documentos como los manuales de clase o las obras de divulgación.

- Artículo científico: Es un trabajo breve (entre 10 y 30 páginas habitualmente) publicado en revistas especializadas. Las características formales de los artículos están definidos por los consejos editoriales de estas revistas. En ocasiones los artículos se publican como capítulos de libro en las llamadas "compilaciones".

- Ponencia: Es la exposición que una persona realiza ante un auditorio, dentro de un evento científico (seminario, congreso, simposium, etc.). Suelen ser trabajos breves a los cuales se concede entre 45 a 90 minutos para su exposición.

- Tesina: Aunque es un término que no está aceptado universalmente, suele designar a trabajos de corta extensión (100 páginas aproximadamente) que se presentan para una evaluación académica dentro de programas de postgrado universitario. Sus características formales dependen de los departamentos o centros donde se llevan a cabo esos programas de postgrado.

- Tesis: Es un documento escrito que una persona presenta en una universidad para obtener el título de doctor. En este documento, el doctorando debe demostrar que domina la materia de la que versa su trabajo así como los procedimientos de investigación en la disciplina donde se encuadra dicha tesis (cuadro 3). Se trata de un trabajo que normalmente requiere la dedicación de varios años, por la complejidad y profundidad de los temas tratados así como por los diseños de investigación que se realizan. Estos trabajos están dirigidos y tutorizados por un doctor en la disciplina. Junto con el documento escrito, la tesis tiene una parte de presentación oral, donde el doctorando realiza una defensa de su investigación ante un tribunal de expertos en la materia. 
- Comunicación: Se trata de una exposición oral que un investigador realiza en un evento científico, a un auditorio especializado y generalmente de número reducido. Las comunicaciones se agrupan por temáticas dentro de estos eventos científicos, de forma que la exposición de las mismas se realizan en una misma sesión, una detrás de otra, dando paso a preguntas y comentarios entre todos los asistentes, bien al final de todas las comunicaciones o tras finalizar cada una de ellas. Estas sesiones suelen tener una duración aproximada de dos horas, dentro de las cuales, cada comunicación dispone habitualmente de entre diez minutos y un cuarto de hora para su presentación. En las comunicaciones se pueden presentar investigaciones concluidas o bien investigaciones que aún no se han finalizado. El objetivo de las comunicaciones es que los asistentes a la exposición ofrezcan sus opiniones y sugieran ideas para mejorar la investigación o para profundizar en sus resultados. Las comunicaciones suelen redactarse y distribuirse entre los asistentes.

- Póster: Se trata de un mural donde se representa, de forma gráfica y textual, los aspectos más destacados de una investigación. Suelen ser utilizados para presentar investigaciones muy específicas o locales. Estos murales quedan expuestos durante todo el tiempo que dura un evento científico, de forma que los asistentes puedan ir viéndolos libremente. No obstante, a los autores de los pósteres se les emplaza durante un tiempo concreto para que los investigadores interesados puedan hacerles preguntas, comentarles cuestiones de interés, abrir vías de continuidad en las investigaciones, etc.

Existen otros formatos que no pueden considerarse trabajos de investigación en sí mismo, pero que sin embargo, tienen una gran utilidad en la práctica de la investigación:

- Reseña: Es un escrito breve (un par de páginas como mucho) que ofrece una revisión crítica de un libro.

- Ensayo: Son libros, generalmente de corta extensión, donde el autor expone ideas, opiniones y razonamientos sin utilizar necesariamente, una metodología científica. Suelen ser obras muy enriquecedoras para la comunidad científica 
porque en ellas se debaten ideas sobre asuntos de actualidad, esbozando cuestiones que suelen inducir nuevas líneas de investigación.

Con relación al uso de gráficos, tablas, ilustraciones, dibujos, fotografías, y figuras entre otros recursos gráficos en los documentos escritos, se pueden tener en consideración algunos consejos ${ }^{2}$ :

- Incluir sólo los estrictamente necesarios.

- No repetir datos que figuran en el texto.

- Deben explicarse bien (con el título y pie).

- Su elaboración debe ser sencilla, sobria, sin adornos ni colores desmedidos.

- Deben estar citados en el texto de forma adecuada.

Con respecto a cuándo incluir estos recursos, se puede tener en cuenta que los esquemas se usan para resumir el diseño del estudio, exponer los instrumentos de recogida y el análisis de datos, así como para mostrar la secuencia de obtención de los resultados. Las tablas y los gráficos se usan, principalmente, para presentar los resultados.

Índice.

Resumen.

Introducción.

Revisión de los antecedentes.

Plan de investigación.

Procedimientote realización.

Presentación de los análisis de los datos.

Comentarios y crítica de los resultados.

Conclusiones.
$10 \%$ del espacio.

$20 \%$ del espacio.

$10 \%$ del espacio.

$15 \%$ del espacio.

$15 \%$ del espacio.

$20 \%$ del espacio.

$10 \%$ del espacio.

\footnotetext{
${ }^{2}$ En el caso del estilo APA, solamente se requería diferenciar entre figuras y tablas.
} 
Referencias y bibliografía.

Anexos.

Cuadro 3. Estructura clásica de las tesis y porcentaje dedicado a cada apartado

\section{Difusión de los resultados}

Para difundir una investigación se puede recurrir a distintos medios, dependiendo de los intereses de los investigadores y de los recursos disponibles para acceder a las editoriales o eventos científicos. Generalmente, las vías más habituales para difundir los resultados de la investigación son los siguientes:

- Eventos científicos: Generalmente congresos, jornadas, forums, etc. En estos eventos los formatos de presentación suelen ser las ponencias, comunicaciones, mesas redondas y pósters.

- Revistas: Los formatos suelen ser artículos, reseñas y resúmenes, entre otros posible, aunque menos habituales.

- Otras vías: Basadas principalmente en las nuevas tecnologías de la comunicación, y por tanto, de reciente aparición. Entre ellos están destacando los blogs y los wikis.

Por último, es imprescindible dedicar, aunque sean dos líneas, a los blogs y wikis. Estos recursos propios de la Web 2.0 son principalmente, iniciativas de grupos de investigación, o de los propios investigadores a nivel individual. En ellos se exponen las líneas de investigación, se presentan los estudios (total o parcialmente), se comentan los resultados obtenidos, etc. El problema de este tipo de literatura es que todavía no existen sistemas de garantía de la calidad de su contenido.
Ed.: edición.
Trad.: traductor.
Ed. rev.: edición revisada.
Cap.: capítulo.
$2^{\mathrm{a}}$ ed.: segunda edición.
Vol.: volumen. 
Ed.: editor.

Eds.: editores.

Comp.: compilador.

Comps.: compiladores.

s. f.: $\sin$ fecha.
Vols.: volúmenes.

No.: número.

Supl.: suplemento

Inf. téc.: informe técnico.

Pte.: parte.

Cuadro 4. Abreviaturas más utilizadas en las referencias 


\section{Anexo}

\section{Normas generales APA para las referencias ${ }^{3}$}

Las entradas con un solo autor se ordenan por el año de publicación, comenzando por el más antiguo:

García, A. (2001). Los indicadores...

García, A. (2002). La pertinencia...

Las entradas con un solo autor preceden a aquellas con varios autores, cuando comienzan con el mismo apellido:

García, A. (2001). Los indicadores...

García, A. y Jiménez, A. (2002). La pertinencia ...

Las referencias con un mismo primer autor, pero con los segundos y siguientes autores diferentes se ordenan alfabéticamente por el apellido del segundo autor y así sucesivamente:

García, A., y Jiménez, E. (2001). Los indicadores...

García, A., y León, E. (2001). La pertinencia...

Las referencias con el mismo autor o autores y con la misma fecha de publicación se ordenan alfabéticamente por el título, siempre y cuando no formen parte de una serie, en cuyo caso se ordenan siguiendo el orden de la serie y no por el título. En todos estos casos se añade una letra en minúscula al año:

García, A. (2001a). Los indicadores...

García, A. (2001b). La pertinencia...

\footnotetext{
$35^{\mathrm{a}}$ edición de APA.
} 
Los trabajos realizados por diferentes autores con el mismo apellido pero distinto nombre, se ordenan alfabéticamente por la primera inicial del nombre. En estos casos en el texto se escriben las iniciales del nombre junto con el apellido.

García, A. (2001). Los indicadores...

García, E. (2001). La pertinencia ...

En el caso de que la autoría sea de una entidad, se dice que estamos ante un "autor corporativo". En estos casos la ordenación se realiza por el nombre completo de dicha entidad.

AIDIPE (2001). Actas del congreso...

Si el trabajo es anónimo, la referencia comienza con la palabra "anónimo" y se ordena como si esta palabra fuese un apellido. Si la obra no es anónima, pero la autoría está en debate, se escribe directamente el título del documento, seguido del año.

Anónimo (2001). Los indicadores ...

Los indicadores del sistema educativo (2001)....

\section{Normas según el medio de comunicación}

1. Publicaciones periódicas (diarios, revistas, boletines ilustrados y otros semejantes):

Autor, A. A., Autor, B. B. y Autor, C. C. (Año de publicación). Título del artículo.

Titulo de la revista cientifica en cursiva, volumen sin utilizar abreviaturas y en cursiva (número entre paréntesis sin utilizar abreviaturas), páginas sin utilizar abreviaturas y separadas por un guión.

Ejemplo:

Jiménez, J.E., Hernández, S., y Conforti, J. (2006). ¿Existen patrones diferentes de asimetría cerebral entre subtipos disléxicos?. Psicothema, 18, 507-513. 
2. Publicaciones no periódicas (libros, monografías, manuales y medios audiovisuales):

Autor, A. A. (Año de publicación). Título del trabajo en cursiva. Localidad: Editorial. Ejemplo:

Campbell, D. y Stanley, J. (1973). Diseños experimentales y cuasi experimentales en la investigación social. Buenos Aires: Amorrotu.

3. Capítulos de un libro:

Autor, A. A. y Autor, E. E. (Año de publicación). Título del capítulo. En A. Editor, E.

Editor y C. Editor (Eds.), Título del trabajo en cursiva. (pp. xxx-xxx). Localidad: Editorial.

Ejemplo:

Zitt, M., y Bassecoulard, E. (2004). Internationalisation in science in the prism of bibliometric indicators. En H. F. Moed, W. Glänzel y U. Schmoch (Eds.), Handbook of quantitative science and technology research. (pp. 407-436). Dordrecht: Kluwer.

4. Para un artículo de diario de noticias:

Autor, A.A. (Año de publicación, mes y día). Título del artículo. Nombre del diario en cursiva, pp. xx, $\mathrm{xx}$.

Ejemplo:

Goleman, D. (1991, octubre 24). Battle of insurers vs. therapists: Cost control pitted against proper care. New York Times, pp. D1, D9.

5. Una ponencia, comunicación, póster, etc., presentada en un evento científico:

Autor, A.A. (Año de realización, mes y día). Título de la presentación. Tipo de presentación en cursiva, Título del evento, Institución donde se celebró, Ciudad de celebración. 
Ejemplo:

De Leeuw, E. (2008, Septiembre 26). Mixed model data collection in surveys. Conferencia, V Congreso de Metodología de Encuestas, Universidad de Córdoba, Córdoba.

7. Una revista en Internet:

Autor, A.A. (Año de publicación). Título en cursiva. Revista. Recuperado Mes día, año de consulta, a partir de dirección web.

Ejemplo:

Dobson, J. (2001). Challenges in the teen years: Understanding early adolescents.

Recuperado junio 14, 2004, a partir de

http://www.focusonyourchild.com/develop/art1/A0000549.html.

8. Películas:

Director, D.D. (Director). (Año de realización). Título en cursiva [tipo de producción]. País de realización: Productora.

Ejemplo:

Bekmambetov, T. (Director). (2007). Wanted [Película]. USA: Universal Pictures. 